Conclusion Our findings suggest that MS but not LVH was associated with the incidence of AF and may be a risk factor for AF.

\title{
[gw22-e0558] METABOLIC SYNDROME AND RISK OF ATRIAL FIBRILLATION IN CHINESE ESSENTIAL HYPERTENSIVE PATIENTS
}

Peng Feng, Lin Jinxiu, Liu Huilan The First Affiliated Hospital Of Fujian Medical University, Fujian, China

10.1136/heartjnl-2011-300867.373

Purpose: Metabolic syndrome (MS) has been associated with a high prevalence of atrial fibrillation (AF) in a community based population. However, there is little data regarding the association between $\mathrm{MS}$ and risk of atrial fibrillation (AF) in essential hypertensive (EH) patients.

Methods This was a retrospective cross-sectional analysis of prospectively collected data in a total of 2782 hospitalised EH patients from 1995 to 2007 in China, which were divided into $E H+$ non-MS group $(n=1761)$ and $E H+M S$ group $(n=1021)$ according to MS criteria. And according to with left ventricular hypertrophy (LVH) or not, EH patients were further divided into four subgroups: non-LVH+non-MS+EH group ( $n=728)$, non- $\mathrm{LVH}+\mathrm{MS}+\mathrm{EH}$ group $(\mathrm{n}=439)$, $\mathrm{LVH}+$ non- $\mathrm{MS}+\mathrm{EH}$ group $(n=1033)$ and $L V H+M S+E H$ group $(n=582)$. The incidence of AF was compared in such groups.

Results From this database there were 1021 patients with MS, and 289 of these patients were diagnosed with AF. The incidence of $\mathrm{AF}$ in patients with $\mathrm{EH}$ and $\mathrm{MS}(\mathrm{EH}+\mathrm{MS})$ group was greater than patients only with $\mathrm{EH}(\mathrm{EH}+$ non-MS). Left atrial diameter (LAD), left ventricular end-diastolic diameter (LVEDD), and left ventricular mass (LVM)were significantly higher in $\mathrm{EH}+\mathrm{MS}$ group than those in $\mathrm{EH}+$ non-MS group. A greater proportion of AF patients also had MS than the patients without AF. Logistic regression analysis suggested age, hypertension duration, LAD, LVEDD, MS, obesity and the use of statins were independent predictors for AF, but not ventricular hypertrophy (LVH). Obesity was the only MS-related risk factors that was an independent risk factor for AF among all the $\mathrm{EH}$ patients in this cohort. 